\title{
EQUALITY OF MINIMAL AND MAXIMAL EXTENSIONS OF PARTIAL DIFFERENTIAL OPERATORS IN $L_{p}\left(R^{n}\right)^{1}$
}

\author{
RONALD ALLEN GOLDSTEIN
}

It is known [1] that if $\Omega$ is a bounded domain, and $P=P(D)$ is a linear partial differential operator with constant coefficients, then every weak solution in $L_{2}(\Omega)$ with compact support in $\Omega$, is also a strong solution.

In this paper, this result is generalized to show that the weak and strong solutions are equivalent for $\Omega=R^{n}$ and $L_{p}\left(R^{n}\right) ; 1 \leqq p<\infty$, without the assumption that the solutions have compact support.

We consider a linear partial differential operator of order $m$, with constant coefficients: $P=P(D)=\sum_{|\alpha| \leqq m} a_{\alpha} D^{\alpha} ; x \in R^{n}$. Here, $\alpha=\left(\alpha_{1}, \cdots, \alpha_{n}\right)$; the $\alpha_{k}$ are nonnegative integers and $|\alpha|=\sum \alpha_{k}$. $D=\left(D_{1}, \cdots, D_{n}\right) ; D_{k}=(1 / i)\left(\partial / \partial x_{k}\right)$, and $D^{\alpha}=D_{1}^{\alpha_{1}} \cdots D_{n}^{\alpha_{n}}$.

The double-barbed arrow " $\rightarrow$ " will denote strong convergence in $L_{p}$, while the single-barbed arrow " $\rightarrow$ " will denote weak convergence in $L_{p}$.

The convolution of two functions, $u$ and $v$, will be denoted by $u * v=\int u(y) v(x-y) d y$.

Unless otherwise specified, all spaces will consist of functions on $R^{n}$, e.g., $C_{0}^{\infty}$ means $C_{0}^{\infty}\left(R^{n}\right)$. Now, suppose $P=P(D)$ has domain $D(P)$, and range in $L_{p}, 1 \leqq p<\infty$. Let us take $D(P)=C_{0}^{\infty}$. It then follows that $P$ is not closed, but satisfies the weaker property of being pre-closed.

Let $P_{0}$ be the closure (in $L_{p}$ ) of $P$ on $C_{0}^{\infty} . P_{0}$ is termed the minimal operator associated with $P$. The maximal operator $P$ is defined as follows. $u \in L_{p}$ is in the maximal domain if $\exists v \in L_{p}:\left(u, P^{*} \phi\right)=(v, \phi)$, $\forall \phi \in C_{0}^{\infty}$. We then say $P u=v$. We prove

Theorem 1. If $P=P(D)$ is a linear partial differential operator with constant coefficients on $C_{0}^{\infty}$ into $L_{p}$, then the minimal and maximal extensions are equivalent for $1 \leqq p<\infty$.

For the proof, consider a function $\psi=\psi(x)$ :

(i) $\psi \in C_{0}^{\infty}\left(K_{2}\right)$; where $K_{2}=\{x:|x|<2\}$.

(ii) $0 \leqq \psi \leqq 1,\left(\forall x \in K_{2}\right) ; \psi(x) \equiv 1$ for $|x| \leqq 1$. Then, form the sequence of functions $\psi^{i}=\psi^{i}(x) \equiv \psi(x / i)$.

Received by the editors April 18, 1966.

1 The research for this paper was supported in part by a Ford Foundation predoctoral fellowship, and in part by a NSF undergraduate research and independent study grant. 
Lemma 1. For each $\phi \in C_{0}^{\infty}$, we have $\left(\psi^{i} u\right) * \phi \rightarrow u * \phi$ in $L_{p}$, for $u \in L_{p}$; $1 \leqq p<\infty$.

Proof. Since $u \in L_{p}$, for each $\epsilon>0$, there exists an $M=M(\epsilon)>0$ :

$$
\int_{|x|>M}|u|^{p}<\frac{\epsilon^{p}}{2} .
$$

Now, choose $i$ large enough so that $i>M$. By Young's inequality,

$$
\left\|\left[\left(1-\psi^{i}\right) u\right] * \phi\right\|_{p} \leqq\|\phi\|_{1}\left\|\left(1-\psi^{i}\right) u\right\|_{p} .
$$

But

$$
\begin{aligned}
\left\|\left(1-\psi^{i}\right) u\right\|_{p}^{p} & =\int_{|x| \leq M}\left|\left(1-\psi^{i}\right) u\right|^{p}+\int_{|x|>M}\left|\left(1-\psi^{i}\right) u\right|^{p} \\
& \leqq 2 \int_{|x|>M}|u|^{p}<\epsilon^{p} .
\end{aligned}
$$

Hence, we have proved the lemma.

Proof of Theorem 1. Suppose for $u, v \in L_{p}$, that

$$
\left(u, P^{*} \phi\right)=(v, \phi) ; \quad\left(\forall \phi \in C_{0}^{\infty}\right) .
$$

Let $j(x)$ denote a function:

$$
j(x) \in C_{0}^{\infty} ; \quad \int j=1 ; j \geqq 0 ; \quad \operatorname{supp} j=\{x:|x| \leqq 1\} .
$$

Let $j_{\nu}=j_{\nu}(x) \equiv \nu^{n} j(\nu x)$, and form the mollifiers of $u$ : $u_{\nu}=u_{\nu}(x)$ $\equiv u * j_{\nu}$. Letting $f^{(x)}(y) \equiv f(x-y)$, we write $u_{\nu}=u * j .=\int u(y) j_{\nu}(x-y) d y$ $\equiv\left(u, j_{v}^{(x)}\right)$. Now substitute $j_{v}^{(x)}$ for $\phi$ in (1) to obtain

$$
\left(u, P^{*} j_{\nu}^{(x)}\right)=\left(v, j_{\nu}^{(x)}\right)=v_{\nu},
$$

the mollifiers of $v$. But, if $u \in L_{p}$, then for any $\phi \in C_{0}^{\infty}$

(3) $P(u * \phi) \equiv P(D)(u * \phi)=(P u) * \phi=\left(P u, \phi^{(x)}\right)=\left(u, P^{*} \phi^{(x)}\right)$.

Therefore, for $u \in L_{p}$, (2) becomes

$$
P u_{\nu}=P\left(u * j_{\nu}\right)=v_{\nu} ; \quad u_{\nu}, v_{\nu} \in C^{\infty} .
$$

Now, apply Lemma 1 to $\phi=j_{\nu}$ and to $\phi=P^{*} j_{\nu}$ to obtain

$$
u_{i \nu} \equiv\left(\psi^{i} u\right) * j_{\nu} \rightarrow u * j_{\nu}=u_{\nu}
$$

and

$$
P u_{i \nu}=\left(\psi^{i} u\right) * P^{*} j_{\nu} \rightarrow u * P^{*} j_{\nu}=v_{\nu}
$$


But $\left(\psi^{i} u\right)$ has compact support, and, hence, $\left(\psi^{i} u\right) * j_{\nu} \equiv u_{i \nu} \in C_{n}^{\infty}$. Now. by (4), for each $\nu$, there exists an integer $I_{1}=I_{1}(\nu)$ :

$$
\left\|u_{i v}-u_{\nu}\right\|_{p}<1 / 2^{v} ; \quad\left(\forall i \geqq I_{1}\right) .
$$

Furthermore, by (5), for each $\nu$ there exists an $I_{2}=I_{2}(\nu)$ :

$$
\left\|P u_{i v}-v_{\nu}\right\|_{p}<1 / 2^{\nu} ; \quad\left(\forall i \geqq I_{2}\right) .
$$

Take $I \equiv I(\nu) \equiv \max \left[I_{1}, I_{2}\right]$; then, setting $S_{\nu} \equiv u_{I \nu}$, we have

$$
\left\|S_{\nu}-u_{\nu}\right\|_{p}<1 / 2^{\nu} ; \quad \nu=1,2, \cdots,
$$

and

$$
\left\|P S_{\nu}-v_{\nu}\right\|_{p}<1 / 2^{\nu} ; \quad \nu=1,2, \cdots .
$$

Therefore, we have exhibited a sequence $\left\{S_{\nu}\right\}$ such that

(i) $S_{\nu} \in C_{0}^{\infty} \equiv D(P)$,

(ii) $S_{\nu} \rightarrow u$; since $\left\|S_{\nu}-u\right\|_{p} \leqq\left\|S_{\nu}-u_{\nu}\right\|_{p}+\left\|u_{\nu}-u\right\|_{p}<1 / 2^{\nu}+\left\|u_{\nu}-u\right\|_{p}$ $\rightarrow 0$;

(iii) $P S_{\nu} \rightarrow v$; since $\left\|P S_{\nu}-v\right\|_{p} \leqq\left\|P S_{\nu}-v_{\nu}\right\|_{p}+\left\|v_{\nu}-v\right\|_{p}<1 / 2^{\nu}+$ $\left\|v_{\nu}-v\right\|_{p \rightarrow 0}$

which proves that $u$ is a strong solution of $P u=v$.

It is interesting that if the spaces are restricted to functions on a bounded domain of $R^{n}$ and a weak solution has compact support $S \subset R^{n}$, then the proof that it is a strong solution in $L_{p}(1 \leqq p<\infty)$ is identical with the short proof of Hormander [1] for $p=2$.

TheOREM 2. Let $P=P(D)$ be a linear partial differential operator with constant coefficients on $C_{0}^{\infty}(\Omega) \rightarrow L_{p}(\Omega)$. Then, if $u$ is a weak solution of $P u=v$ such that supp $u=\delta \subset \Omega, u$ is also a strong solution for $1 \leqq p<\infty$.

Proof. For $u_{\nu}=u * j_{\nu}$, we have $u_{\nu} \in C_{0}^{\infty}(\Omega) ; \nu>1 / \lambda$, where $\lambda$ is the distance from $s$ to the complement of $\Omega$. Then, $P u_{\nu}=\left(u, P^{*} j_{\nu}^{(x)}\right)$ $=\left(v, j_{\nu}^{(x)}\right)=v_{\nu}$. Hence, $u_{\nu} \in D(P), u_{\nu} \rightarrow u$, and $P u_{\nu} \rightarrow v$.

I wish to gratefully acknowledge the invaluable assistance I received from Professor Martin Schechter, who, through his advice and teaching, presented me with this problem and the knowledge necessary and sufficient to cope with it.

\section{REFERENCES}

1. L. Hormander, The theory of general partial differential operators, Acta Math. 94 (1955), 161-248.

2. M. Schechter, L. Bers and F. John, Partial differential equations, Interscience, New York, 1964.

NeW YoRK UNIVERSITY 